\title{
Connective and Communal Public Goods in Interactive Communication Systems
}

\footnotetext{
This paper extends theories of public goods to interactive communication systems. Two key public communication goods are identified. Connectivity provides point-to-point communication, and communality links members through commonly held information, such as that often found in databases. These extensions are important, we argue, because communication public goods operate differently from traditional material public goods. These differences have important implications for costs, benefits, and the realization of a critical mass of users that is necessary for realization of the good. We also explore multifunctional goods that combine various features and hybrid goods that link private goods to public ones. We examine the applicability of two key assumptions of public goods theory to interactive communication systems. First, jointness of supply specifies that consumption of a public good does not diminish its availability to others. Second, impossibility of exclusion stipulates that all members of the public have access to the good. We conclude with suggestions for further theoretical development.
}

Public goods theories grapple with the age-old problem of how to induce collaborative problem solving and other forms of collective action among self-interested individuals, groups, or organizations, assuming, of course, that they share at least some common goals. When successful, such collective action generates so-called public goods, such as parks, roads, libraries, neighborhood brush removal for fire prevention, beach cleanups, or other organized collective goals. Inducing collective action for interorganizational efforts is also a formidable challenge, applied in such diverse arenas as the United Nations, business cartels, conglomerations of charitable organizations, the Japanese keiretsu, and health service provider networks.

Possibilities for collective action have expanded with recent advancements in information and communication technologies such as electronic mail, cellular telephones, and fax machines as well as the increased availability, complexity, and linkages of database systems, electronic bulletin boards, and other public and private information forums. These new capabilities can, in certain contexts and with appropriate inducements, support electronic communities such as the City of Santa Monica's Public 
Electronic Network. This network provides an electronic forum for residents to share information directly among themselves, as well as a mechanism for input to city policy formulation (Schmitz, Rogers, Phillips, \& Paschal, 1993). Yet, new technologies can also fail to deliver the expected set of linkages, such as in the case of the residential video telephone.

At the organizational level new technologies can support collective action through information-sharing capabilities that transcend organizational boundaries. Badaracco (1991) referred to such interorganizational information-sharing networks as "knowledge links" (rather than "product links"). Recent examples include the microcomputer consortium of universities, the Sematech venture in the electronics industry, and attempts to develop an information clearinghouse of community resources for rebuilding Los Angeles after the 1992 riots. As the relative lack of success of the latter two examples indicates, these collaborative interorganizational efforts are often difficult to launch and even harder to sustain. Key impediments to such collective action frequently lie in inducing organizations to jointly supply the information in service of mutual interests and to employ the technological platforms that permit collaborative information processing.

These concerns point to a significant opportunity for studies of collective action. Public goods theory can be extended to explain the conditions associated with the relative success or failure of interactive communication system linkages among individuals. However, most of the theory and research on collective action has focused on traditional material public goods such as parks and bridges (e.g., Barry \& Hardin, 1982; Marwell \& Oliver, 1993; Oliver, Marwell, \& Teixeira, 1985; Olson, 1965; Samuelson, 1954). To date, information and communication forms of public goods have received very little attention. This omission is crucial because information and communication systems possess two features that distinguish them from material forms of public goodsconnectivity and communality. We argue in this article that these two unique features lead interactive communication public goods to behave differently than traditional material goods in the collective action context. This, in turn, implies that several modifications are required to develop a useful theory about interactive communication public goods.

The purpose of this article is to further advance recent extensions of public goods theory to the realm of interactive communication systems (Connolly \& Thorn, 1990; Markus, 1990). First, we review "public" goods theory and recent applications to information and communication systems. Second, we develop the concepts of connectivity and communality as special forms of public goods. Third, we examine the assumptions underlying the theory and propose several modifications that are appropriate for interactive communication systems. Finally, we raise several issues that stem from this work and propose future lines of development.

All theoretical development, including the present effort, requires the 
explication of concepts that are necessarily abstract. Therefore, to link these abstractions to concrete instances where interactive communication systems are critical to successful collective action, we provide a series of examples. These examples are selected to illustrate a variety of different communication systems. In order to provide some continuity and integrative thrust one example is also carried throughout the discussion. We begin with a background for this example and then turn to our explication of the central tenets of public goods theory.

Collective action through interactive communication systems offers the potential for substantial benefits in many areas of significant public concern such as law enforcement. Efforts to control the importation, distribution, and sale of illegal narcotics have been severely hampered by the lack of cooperation across federal, state, and local levels of the criminal justice community (Bureau of Justice Statistics, 1992). This fragmentation of efforts, and occasional conflicts over jurisdiction, adds to the already serious concern that organized illegal drug cartels are making significant advances by using interactive communication technology. Multijurisdictional alliances among antidrug organizations can offer significant counterpressures (Chaiken, Chaiken, \& Karchmer, 1990). Advanced information and communication systems provide a platform for information sharing that can support collective police action and can match the sophistication and communication technology used by the drug cartels. The key is to induce criminal justice organizations to work together and share information (Bureau of Justice Statistics, 1992). However, the sharing of privileged drug enforcement information by officers entails potentially significant costs, including officer safety, that can serve as disincentives to truly collective efforts.

\section{Theoretical Approaches}

The concept of the public good has a long history, mostly in Continental philosophy. John Locke, David Hume, Adam Smith, and John Stuart Mill all addressed the concept to varying degrees. In this section we first review the foundations of public goods theory. Then we examine two recent theoretical extensions to interactive media and discretionary databases.

\section{Public Goods Theory}

The modern discussion of public goods is derived from economic theory and Samuelson's (1954) formulation. The defining characteristics of a public good are jointness of supply (Barry $\&$ Hardin, 1982) and impossibility of exclusion (Head, 1972). Jointness of supply stipulates that "one person's consumption of it does not reduce the amount available to anyone else" (Hardin, 1982, p. 17). It is difficult to think of a physical good that truly is held in joint supply. For example, although one's use of a public park generally does not diminish another's enjoyment of it, there may be occasions when the number of visitors to the park reaches 
a point where no more visitors can be accommodated. In such a case, parks exhibit "crowding" (Barry \& Hardin, 1982, p. 196) and one's consumption of the good effectively reduces the amount available to another. Chamberlain (1974) noted that most public goods will suffer from crowding and thus few goods are purely joint in nature. Even clean air, which arguably was held in joint supply prior to modern industrialization, can no longer be said to be held jointly. Certainly there are enough people and organizations who consume clean air today in such a manner that its supply is greatly altered, even reduced, for others. Samuelson's (1954) formulation recognized this general problem, but as his focus was designed to describe the "pure theory" of public goods ( $p$. 387 ), the rarity of perfectly joint goods was not considered an impediment. Head (1972) and Chamberlain (1974) subsequently focused on relatively rather than perfectly joint goods.

Impossibility of exclusion from a public good stipulates that "it is impossible to prevent relevant people from consuming it" (Barry \& Hardin, 1982, p. 31). Within the public under consideration, all members have an opportunity to benefit from the good. For example, members of the relevant public cannot be denied access differentially to the public good of a public park or road, except in cases of emergency. Chamberlain (1974) offers a more refined definition by pointing out that "individuals who do not share in paying for the good cannot be excluded from enjoying the benefits of the good. The benefits accrue automatically to all individuals" (p. 707).

Size of the public and the free-rider problem. One of Samuelson's (1954) key postulates was that spontaneous provision of a public good by the aggregate desires of decentralized populations was impossible. Some form of central authority is necessary to provide the good (p. 389). Olson (1965) explored this idea by developing a "logic of collective action" which combined public goods theory with Von Neumann and Morgenstern's (1944) game theory. Olson's method was to view the provision of collective goods as an $n$-player, iterated prisoner's dilemma game. A key issue related to willingness to contribute is individuals' perceptions of whether their individual contributions could make a visible difference in the level of the good. The principle finding was that large groups would tend to fail in obtaining a public good because individual contributions were not very noticeable; conversely, small groups would succeed because contributions were more noticeable. Subsequent investigations have focused primarily on this "size problem" (Chamberlain, 1974, Hardin, 1982). Critics of Olson's claim (e.g., Oliver \& Marwell, 1988) argue that high jointness of supply makes cost unrelated to group size; therefore, size may be positively related to noticeability of contributions and the likelihood of collective action (Marwell \& Oliver, 1993; Oliver, 1993).

A derivative of the size issue is the "free-rider problem" (Barry \& Hardin, 1982; Sweeney, 1973). Free riders are those who enjoy the 
benefits of a public good without contributing to its establishment or maintenance. Sweeny (1973) defined a free rider as one who "will perceive his efforts to be unnecessary and will withhold part of his contribution knowing that the efforts of others will be sufficient to achieve the goal" (p. 279). Theoretically, free riders are more likely in large groups, where contributions are less noticeable and benefits are divided more broadly (Olson, 1965). However, high jointness of supply in large groups, as noted above, may serve to reduce these tendencies (Marwell \& Oliver, 1993).

Interest and resource heterogeneity. Another key concern of public goods theory is the heterogeneity across members of a community. This heterogeneity takes two forms: (1) peoples' interests in achieving the good, and (2) the resources they can bring to bear to make it happen. Ironically, greater heterogeneity can favor collective action when the mean level of interest or resources would otherwise produce failure, or can inhibit collective action when the mean level would have produced success (Oliver, 1993, p. 285).

Consider the former case, when the mean level of collective contributions is deficient. Variation in a population increases the likelihood that there will be at least some individuals who are interested or resourceful enough to find ways to develop the good (Oliver, Marwell, \& Teixeira, 1985). For example, although most people want fresh lakes and unpolluted oceans, those who want them most will be swimmers, boaters, and divers. These persons will be most motivated to take action to ensure that we have them. Similarly, although most members of a community may want legislation to ban polluting their water resources, those who have the greatest political clout will have the greatest likelihood of securing the protective legislation for the whole community.

On the other hand, heterogeneity can stand in the way of collective action even when mean levels of interest or resources appear sufficient. Collective action that restricts an individual's contributions to within narrow ranges should be especially prone to this problem. As variation in the population increases, the interests or resources of some individuals fall too low for other individuals to make up. One example is a fair democratic election. Individuals are restricted to casting a single vote and must make a certain minimum investment of time and effort to do so. Suppose in a particular election that the mean level of resources (e.g., time, transportation to a polling place, etc.) is sufficient for everyone to vote, but in fact that a majority of the eligible electorate cannot make it to a polling place. The government would fail to gain legitimacy through a fair election. The most that a few wealthy individuals could do is to attempt to make the polls universally accessible by financing the election more amply. Casting the actual votes is a collective action that demands broad participation by members of the public in order to succeed.

\section{Critical Mass Theory of Interactive Media}

An interesting recent extension of traditional public goods theory is Markus's (1990) critical mass theory of interactive media. Markus's theory 
focuses on a public good in the form of universal access to an interactive communication medium. The universal access good is realized when each member of the public of interest (what Markus terms "community") is able to communicate with every other member via the medium (e.g., universal access to the public of a nation via a national telephone system). A key issue in the development of universal access is achieving critical mass - a sufficient number of individuals willing to pay the costs of adopting a medium in the absence of universal access. Without a sizable number of communication partners available through it, individuals are unlikely to adopt the technology and implementation will fail (Markus, 1990, p. 196). Thus, the likelihood of success for a new interactive medium is linked to reciprocal interdependence across adopters and potential adopters. This interdependence is particularly problematic because early adopters can elect to return to nonadopter status if they judge that not enough others have adopted to make continued participation worthwhile. ${ }^{2}$

Markus (1990) argued that community size is related in complex ways to the realization of universal access. Large communities are more likely to realize universal access when a substantial infrastructure is required or when benefits continue to increase with the number of users. Small communities, on the other hand, should be favored in those cases where little infrastructure is required, because costs are proportional to the number of users. Markus also accepts the premise of public goods theories where heterogeneity of expected benefits and contributory resources facilitates realization of the good.

Three key factors are important to realize universal access to interactive media (Markus, 1990, pp. 206-208). Task interdependence in taskbased communities implies heterogeneity because it often results from functional specialization. Specialization creates pockets of information resources that are more accessible within than outside the specialist subgroup. Centralization of resources promotes universal access because resources are more unevenly distributed in centralized communities, which tend to be functionally specialized, as compared to decentralized communities where each node must have a sufficient variety of functional resources to operate fairly independently. Geographic dispersion of information sources implies that some sources will be more distant than others. The more distant sources will be more attracted to interactive media as a means of communicating vital information.

\section{Discretionary Databases as Public Goods}

An alternative extension of public goods theory focuses on a type of information public good that Connolly and Thorn (1990) termed discretionary database: "a shared pool of data to which several participants (individuals, departments) may, if they choose, separately contribute information" (p. 221). Such databases are most valuable when they contain useful and timely information. The key problem is that the payoff function for individual contributors rewards withholding rather than contributing information, because each contribution benefits all users 
except the contributor, who already has the information. Such information goods, then, are likely to be undersupplied.

Countervailing incentives can reduce the cost of contribution and increase the payoff to contributors. An organization might subsidize contributions, such as providing data-entry equipment and personnel, training on system use, or awards for contributions. However, when incentives are greater than costs, persons will be tempted to contribute volume rather than quality of information, producing an oversupply of information, not all of which may be valuable. Consider the example of the interorganizational drug enforcement information system mentioned earlier. One inducement to contribute information to an ongoing drug investigation provided by current federal law is that a portion of assets seized from drug criminals and subsequently sold by the government is returned to the participating law enforcement organizations. The value of these seized assets (e.g., cash, bank accounts, houses, boats, airplanes, and any other asset used in illegal drug activity) can be quite substantial, running as high as millions of dollars. To the extent that a participating organization's share of asset seizures is based on the volume of information it contributed to the investigation, there is an incentive not to screen out irrelevant, redundant, or low-quality information.

According to Connolly and Thorn (1990), the motivational mechanism in operation for contributions to a discretionary database is the norm of reciprocity. Individuals will contribute to the extent that they believe their contributions will be reciprocated by others. The reciprocation mechanism has implications for both optimal group size and the effects of heterogeneity of interests and resources. First, small systems will induce greater contributions, since the reciprocal link between contributor and user will be more visible. Second, inequalities (heterogeneity) across users in information quality, cost, or benefit will reduce contributions, since reciprocity is more difficult to obtain when resources possessed by the different contributors are not equal. This theory differs from other public goods formulations that predict greater likelihood of collective action under conditions of heterogeneity of interests and resources. Nevertheless, both Connolly and Thorn's experimental study (1990) and Rafaeli and LaRose's (1993) survey of users of public bulletin boards found Connolly and Thorn's predicted patterns with respect to both group size and inequalities.

\section{Connectivity and Communality as Public Goods in Interactive Communication Systems}

Markus's (1990) critical mass theory of interactive media and Connolly and Thorn's (1990) formulation of discretionary databases illustrate two distinct classes of public goods involving interactive communication systems. The systems themselves are not public goods. Rather, the public goods are the communication and information functions that the systems can provide. In this section we distinguish two general function-based 
classes of information and communication public goods: connectivity and communality. Universal access discussed in the prior section can be viewed as an outcome arising from provision and use of an interactive media system based on a connective public good. A discretionary database is shared information accessible to all members who participate in a communal public good.

\section{Connectivity}

Connectivity fulfills one of the most basic functions of communication to link members together (McQuail, 1987, p. 331). Markus's (1990) universal access falls within the broad class of connective public goods. Connectivity involves direct communication among members of a public. Full connectivity rests upon the creation of a jointly held system that provide all members of the public with the means to communicate with each other. Full connectivity has been a central concern in the development of a wide variety of communication systems, such as the federal postal service at the national level and the public bulletin board at the community level. Full connectivity needs are also evident in the development of transportation systems, such as extensive networks of roads and railroads for land-based connection and complex route structures for air or sea travel. The underlying rationale for full connectivity is that the public's good is secured, in part, by the ability of all of its members to communicate privately, associate freely, and link easily with any subgroup of persons or other entities (e.g., businesses) as they see fit through the use of common carriers.

The capability for full public connectivity creates a vast potential for direct contact between members that may not materialize in practice. Telephone usage illustrates the point. Although each user could call any number at will and expect an answer of some sort, no single user ever does call them all. Characteristic of normal telephone usage is a large number of relatively small, overlapping, and often shifting coalitions of users who contact each other directly. The more unpredictable the need for direct connection between specific user nodes, the more valuable is full connectivity. A preexisting infrastructure minimizes the barriers to making a new social contact whenever the need arises.

For a given technology and user group, the most economically viable system might not be fully connected, if social contacts are sufficiently predictable and slow to change. The marginal cost of adding new connections in the event of changing demand could be less than providing, in advance, for all possible connections. A system that provides less than full connections may nevertheless offer optimal connectivity. An optimally connected system's long-term viability depends on how responsively it matches the dynamic pattern of social relationships by providing the fullest connectivity desired.

\section{Communality}

A second class of public goods attained through communication is communality, where members jointly hold a single body of information. Communality is the true good underlying discretionary databases. Com- 
munality rests upon the creation of a forum for collectively sharing information. Each member may draw upon this store and at least some must contribute to its maintenance. Examples of information systems that provide communal goods include census records, trade association statistics for an industry, a city library, or a company bulletin board.

Communality provides for a wider variety of information exchanges than connectivity, or the direct exchange of information between pairs of individuals. In addition to direct exchange, communality facilitates generalized and productive types of exchange (see Cook, 1991, pp. 3738). Direct exchanges are still possible, as Connolly and Thorn (1990) emphasized in their discussion of discretionary databases. Systems of this sort function as information marketplaces, helping sellers and buyers (or producers and users) to locate each other through the information they contribute to a common pool. Participants' ultimate purpose is to proceed toward direct, dyadic connections.

By contrast, generalized exchange is a pattern of social exchange where an individual may contribute to and receive resources from different people. As an illustration, suppose that 100 people donated books to create a public library. Each also borrows books from the library. It is unlikely that Person A would borrow only books donated by Person B, and vice versa, so that only dyadic exchanges took place. More likely is that a subset of people would be interested in books donated by Person A while Person A would be interested in books donated by a different subset of people. Any sense that donations are reciprocated would be distributed across a number of individuals or, most likely, mediated by a sense of membership in the collective (Ekeh, 1974). A discretionary database could function in this way, too.

Information sharing sometimes also produces more than the piecemeal exchange of divisible information units. Instead, information is assembled and analyzed to create something new. Census records and industrial statistics exemplify how information can be combined into a new product that is more than simply the sum of its parts. The national patterns that emerge from these data establish a basis for formulating broad public policy or business strategy. This is productive exchange, where members of the relevant public benefit from the communal product they make possible by their contributions of information. As in the case of generalized exchange, a norm of direct reciprocity is of little relevance since dyadic exchange is not evident. Rather, other norms are liable to govern judgments as to who is or is not free riding (e.g., equity; see Stolte, 1987).

Although information exchange via technology is the focus of the present article, communal information need not be mediated. A face-toface group meeting has the purpose of providing communality, where private sidebar discussions typically violate the norm of including everyone in the discussion. Moreover, a collective's symbols, sagas, and the forums by which they are sustained represent communality. The essence 
of communality is found in the forging of a collective identity and purpose. Theorists including Bormann (1983; symbolic convergence) and Habermas (1979; public sphere) have focused on the circumstances wherein this occurs and its necessity to the welfare of any public. Thus, the communal good need not be based in such tangible media as a written or electronic text, for example, even though in many cases such media contribute to accessibility and, therefore, to broad and timely public participation.

\section{Realization of a Connective Public Good}

Costs and benefits impact on the ability to realize a connective public good. To examine these issues we first distinguish two dimensions of connectivity.

Physical and social connectivity. Connectivity has two components. First, physical connectivity among the nodes is realized by the infrastructure that makes direct communication possible. Examples of physical connectivity include the miles of telephone wire and attendant switching hardware for the telephone system, the network of roads, railroads and air and sea routes that makes possible the physical movement of mail and packages, earth- and space-based radio transceiver systems that provide linkage without wires, and the computer and telecommunications systems that make electronic mail feasible.

The second component is the social connection, the participation of the public in the use of the physical connection. Connectivity in a social sense could not be provided by the postal system in the absence of people using it to send materials to each other. The connectivity potential of electronic mail fails when people do not use their accounts. Physical connectivity is a necessary but not sufficient condition for connectivity in a social sense.

Social connectivity is different from physical connectivity in another very important respect. With physical connectivity, one or more persons can provide the infrastructure for all, thus ensuring creation of the good. For example, an organization might provide the hardware for an electronic mail network, and a subset of users might generate the necessary software and set up accounts for everyone. These few actors could provide the opportunity for all to participate. The other members are free riders in the sense that they have access to physical connectivity through an infrastructure that they did not help to provide.

With social connectivity, a few individuals cannot provide the good for the collective; social connectivity is generated by the active participation of each member in the use of the physical network. Whereas a public park could exist as a public good even if few persons actually used it, a public electronic network would most likely fail in providing social connectivity if only a few persons used it. The success of the telephone system as a means of connectivity depends on the actions of individuals to receive, answer, and initiate telephone calls. Thus, social connectivity is a critical input to the ongoing feasibility of interactive communication 
systems. Existence of a physical infrastructure with excellent capacity for linking the relevant public cannot by itself guarantee provision of the connective benefit, a fact system designers often overlook. A common explanation for the failure of even technologically superior electronic mail systems is lack of participation by the target users (Panko, 1984). Furthermore, social connectivity cannot be achieved by free riding in the same sense that a free rider can obtain the benefit from a public park. A person cannot directly benefit from an interactive communication system without being connected. Thus, persons who drop out of contributing by withdrawing their social contributions are not free riders. Since they are no longer connected, they cannot achieve the benefit.

Costs of contribution. Each type of connection, physical and social, has associated with it different kinds of contribution costs. Costs for physical connection include such things as hardware and software, as well as whatever negotiations are required to get a communication system accepted by those who will administer it. The primary costs will be in the initial creation of the physical connection, with some ongoing maintenance costs. Political and financial resources will be applied as contributions.

There are several ways to cover these costs. One way is for a subset of the public to contribute the physical system for the whole, including both the centralized equipment and any local devices required by individual users (e.g., the terminal or computer access to an electronic mail system). An alternative is for a subset to provide the centralized infrastructure, while users provide local connective devices at their own gateways. Finally, users can be asked to subsidize the full cost of both central and local physical connections. Consider our example of the attempt to create a law enforcement consortium. A law enforcement agency with access to a large sum of funds from asset seizures, grants, or other sources could elect to provide the necessary hardware and software for the whole consortium. Alternatively, such an agency might provide only the central system, and require each local agency to assume the costs of the local terminals needed for access. The third alternative would be to require each agency to contribute some portion of the cost for the central system, as well as assume the costs of its own local access devices.

The latter two alternatives involve varying degrees of costs to all members of the public. In all three cases heterogeneity of interests and resources should still facilitate development of the good, because it indicates the likelihood that a subset of persons will exist who can either provide the physical connections or provide incentives for individuals to contribute them (e.g., subsidies for access devices). This latter situation is similar to the early days of the telephone system, where local wiring, handsets, and switching were provided at artificially lower rates for consumers. The cost for this equipment was subsidized by higher charges to business and long-distance customers.

Social connections require an entirely different set of costs, related 
to individual users maintaining an open gateway. Costs include both subjective and objective factors (Marwell \& Oliver, 1993). They include learning how to use the system, giving up established ways of doing things, developing and maintaining the interactive social networks required to use the system, and compiling information to provide to one another. Markus (1990) also described the need for "communication discipline"-making oneself available by sending and receiving communications. The cost of making oneself accessible cannot be borne by anyone other than each member of the public, although resource-rich persons might obtain assistance in sending and replying to communications through support staff. Certainly no subset of contributors can provide social connectivity for all other members of the public. Also, the costs of social connection will be ongoing rather than skewed toward the early phases. This is particularly problematic because adopters can return to nonadopter status. Subsequently, since costs continue, even the realization of universal access can be threatened.

Costs of optimal connectivity. An impetus for the maintenance of less than full connectivity is the high cost of some potential users which results in their exclusion from the system. Members of a community, say residents of a city or employees of an organization, may reasonably expect a particular system to include them all. Yet, certain user nodes might be unusually expensive to connect (e.g., those in physically remote locations), or some nodes may be so underused that they seem to contribute too little to justify their expense. Users who account for the most overpriced or underused connections look like free riders to other users. Excessive creation or maintenance costs attributable to certain user nodes can threaten the entire system, straining the public-spiritedness of full connectivity.

Again, there are several ways to resolve the problem of covering costs for the public good. The first is to distribute costs evenly across contributors, using some connections to subsidize others, such as flat monthly fees for local telephone connections regardless of call volume. A second is to require certain users to make greater contributions to offset higher costs, such as commercial and long-distance customers paying higher rates to subsidize local service. A third is to exclude some people whose inclusion costs too much, as with the lack of express mail services to some remote locations.

Only the first two options maintain a fully connected network. The exclusion of members based on cost effectiveness backs away from the idea of full connectivity as a public good. There is, however, no single definition of "the public" and, consequently, no reason that public goods theory might not apply where practical constraints restrict membership. A user public might comprise almost any group of persons, and their number can shrink or swell over time according to their joint success in maintaining a system that provides its members with a high degree of connectivity. Only when members of a certain predefined public insist 
that their exclusion violates sound public policy does the issue of fixed boundaries become an important issue. A recent example is the case of private global networks accessible exclusively to corporate interests (Sirbu, 1992).

Benefits. Any interactive medium operates in the context of other media that may influence progress toward full connectivity. The existence of a regularly upgraded telephone network that has realized universal access, for example, greatly facilitates electronic mail and fax traffic. Existing telephone lines provide necessary physical connection for these technologies. Were these new technologies required to start from scratch, universal fax or electronic mail access would be much more difficult to achieve.

For social connections, the situation is more complex. The ability to reach any other member of the public by multiple media (e.g., telephone, fax, computer-to-computer connections, postal system) reduces the interests in connectivity via new media in a community. This substitutability across interactive media also reduces the imperatives for universal access through the new medium and contributes to the viability of coalitions of users in the absence of universal access. (The existence of viable coalitions is described in detail later.) Substitutability is likely to work primarily against universal access for a new medium. However, in special circumstances the availability of complementary media may have the opposite effect, facilitating use of a new medium if it can be treated as one aspect of a multimedia system. Consider the ease with which the same textual message in a computer file can be sent via electronic mail or a fax modem. An individual might be willing to learn how to send the text through electronic mail to those who have accounts but no fax-receiving capabilities, since the same file can be sent via fax modem to others of the public who have not adopted electronic mail but who have fax-receiving capabilities. That is, persons may be willing to experiment with a new form of connection when there are other media that connect with nonadopters, so that in combination the multimedia system is fully connected.

With the exception of such special circumstances, the net effect of substitutability is that the new medium must provide better or cheaper connectivity on important dimensions to users in order for them to be willing to bear the social costs of personal adoption. Because connectivity is not unique, critical mass may develop more slowly, if at all, and universal access will be difficult to achieve.

\section{Realization of a Communal Public Good}

Distributed versus clustered resources. Imagine a dating service in which the potential dates did not reveal any information about themselves for the communal record. The administrators of the system might be able to track down basic demographic information on subscribers (age, race, etc.) from various public records or previous dates, but the information that other subscribers would find most useful is that which 
individuals could uniquely provide about themselves. The success of such endeavors rests heavily on the willingness of each subscriber to contribute personal information to the database. When information resources are distributed, participation in the information system by each member is a necessary condition for the success of the communal endeavor. We have seen for social connectivity that members of the public control gateways that determine whether or not their node is connected. Similarly, to the extent that individuals hold private information not available through other sources, they control gateways that affect whether their unique information will contribute to creating a communal good. No subset of individuals can create the communal good for the benefit of the whole. A wealthy individual cannot donate shared information for a database in the same way as land for a public park.

Connolly and Thorn (1990) pointed out that incentives in such a situation work against the viability of a database. Each person who contributes receives no direct benefit from the contribution, since the contributor already knows the information. This contrasts with contributions for connectivity, where an individual may seek to secure direct benefit from establishing communication with another person.

Under the alternative scenario where information is clustered and variably distributed such that some persons control the bulk of it (more centralized information resources), a communal public good might be created for the benefit of all by the actions of an interested and resourceful subset of members. In this situation, the good more closely resembles a traditional public good. Its viability depends on motivating those who have access to the needed resources to employ them in the creation of a good that will benefit everyone. For example, leaders in a particular industry might collect and distribute information about the actions of global competitors to induce other domestic competitors to behave in ways that will maximize the survival of the domestic industry in the face of foreign competition.

Communality costs. Communal databases have physical costs associated with accumulation, storage, and transfer of information. These costs include the knowledge and skills for entry, manipulation, and withdrawal of information. They are theoretically similar to the infrastructure costs for connectivity and can be provided in the same alternative manners. However, the key issue for communal goods is initiating and sustaining information contributions. Costs sometimes involve sharing proprietary information so that it becomes a communal rather than a private possession. In theory, information is held in joint supply because consumption does not reduce the amount available (Barry \& Hardin, 1982). It is possible to share information with another and yet have no less of it as a result. In practice, however, the value of information often changes when individuals share it. For example, sharing information that provides a competitive advantage may decrease its utility to the person who once held it exclusively. Or, the value of information may 
increase if its revelation permits it to be linked with related information that is more useful if employed in combination rather than separately. An extended discussion of the utility and value of information is beyond the scope of this article. These examples simply illustrate that contributions can have costs and benefits to individuals related to how information is transformed by passing from private to public status.

Benefits. If the benefits of creating a communal database are unevenly distributed, some individuals will be more motivated than others to ensure the necessary contributions (heterogeneity of interests). For example, a region that has a particularly high incidence of a serious disease will be willing to contribute substantial resources to the creation of a national database relevant to the etiology of this disease, in the hopes of eradicating it. In the presence of asymmetries of benefits such a database is likely to be created even where information is widely distributed (e.g., in the hands of individual physicians), because those who have most to benefit will create the incentives needed to prompt those who hold the information to contribute it to the communal good. For example, in the 1980s the Katmandu valley region of Nepal faced a serious local epidemic of meningitis. Local officials secured a donation of computing equipment from Apple Computer Corporation that was distributed nationwide to physicians to support the transfer of case information to the communal record. They were ultimately successful in eradicating the disease (Fulk, Von Glinow, \& Rogers, 1988).

Where benefits are more evenly distributed, Connolly and Thorn (1990) predict increased likelihood of developing the public good, because reciprocity will be more readily obtained. However, an alternative rationale would argue that an even distribution of benefits decreases the likelihood that there will be a subset of highly motivated potential contributors. In the absence of the outbreak of the disease noted above, who will be sufficiently motivated to ensure the creation of the database? As we describe later, an overarching administrator who manages contributions can be an important factor in assuring the development of a public good. Governmental bodies often serve this role. In practice it is difficult to imagine many situations of truly symmetrical benefit across all sectors of the relevant public.

Communality, connectivity, and information in the public interest. Connectivity involves a point-to-point transmission, regardless of the public or private origin of the content that is transmitted, and regardless of whether and how the communication is mediated. Communality involves transmission of privately held information to the public, and reception of communal information from that public, also whether it is mediated or not. Connectivity arises from direct point-to-point linkage, whereas communality derives from links through a common forum such as a database.

Communality helps to overcome two difficulties inherent in point-topoint systems. The first is the need for contributors to predict who might 
benefit from a particular set of information. (Distribution lists are a type of viable coalition in a communication system that serve this same purpose, as described later.) It potentially obviates the need for direct connection between the parties and provides the information-dissemination benefits of a fully connected system without the cost of direct connections among all users. Each member need only be physically and socially connected to the communal good. For example, posting a notice on a public bulletin board avoids both the need for the poster to know which specific individuals might value this kind of information and the costs of directly distributing the information to those individuals pointto-point (e.g., time investment, copying, and transmission costs such as postage or log-in time in the case of an electronic bulletin board).

The second difficulty that communal goods overcome is that of trying to anticipate what specific information is needed by other specific users and what synergistic combination of information might result from the contributions of multiple originators. In one sense, the communal database serves the function described in Cohen, March, and Olsen's (1972) "garbage can" model of decision making. It is a receptacle of diverse information, solutions, problems, and criteria. As such, users may select from it the information that best suits their specific needs, and contributors need not know or attempt to predict what those needs might be.

A potential problem in a database system occurs if too much of its information proves irrelevant to the needs of the users or the common interest. On the other hand, a system whose purpose is to provide a general information resource can offset this problem with filtering and search techniques. Databases commonly provide such features as menu and seek functions in order to enable users to search for and select only the information useful to them. Finally, if information relevance is generally narrow and users can be certain who should receive their information, then a point-to-point communication system is a preferable solution to a database.

\section{Dynamics and Multifunctional Goods}

In the case of interactive communication systems, costs and benefits of the connective or communal public good are particularly dynamic due to the continual advancement of electronic technology and the unpredictable quality of users' information needs. On the technological side of the equation, costs are in constant decline for key information processes such as storage, retrieval, data bandwidth (i.e., speed), and link constitution across physical distance. Costs are also saved through the progressive integration of once-separate information processes. Perhaps the most important example is the gradual merger of computing and communication (Bradley, Hausman, \& Nolan, 1993). Clear distinctions between information and communication systems are already beginning to lose meaning. On the human side, exactly who will want to communicate with whom, and when, can be very difficult to predict over the long term. Also unpredictable in many cases is the exact value that any one 
piece of information will have to any one individual. The relative benefits of connectivity versus communality stem mainly from the capacities of systems to cope with these uncertainties.

As a consequence of the convergence of computing and communication, systems now increasingly provide for both connective and communal functions, often in the context of still other capabilities. A common example is the personal computer connected to an electronic mail network and to a computer bulletin board. The same system (as seen from the user's standpoint) provides both a connective and communal good.

Multifunction goods may be sustained on the strength of a single function (connective or communal). Once a primary function has reached critical mass, the marginal cost of the auxiliary function may be relatively low in comparison to its cost were it a primary function. Thus, the threshold for success (i.e., the critical mass of users) on auxiliary functions can be much lower than would otherwise be the case. Returning to the previous example, if the personal computer network in a given business is cost effective solely on the basis of its communal function as a joint information base for accounting purposes, only a small proportion of users corresponding via electronic mail might justify the added expense of that connective function. And, if more users can be motivated over time to employ electronic mail, the fully connective physical infrastructure is already in place. Multifunctionality makes it possible to separate physical and social contributions for the auxiliary functions in ways not possible for primary functions or for unifunctional systems. For example, the newly emerging electronic bill-paying services are likely to grow rapidly over time on the basis of an existing infrastructure of connectivity for electronic funds transfer in combination with the increasing number of modems already in the hands of computer users for the purpose of electronic mail and information services.

\section{Issues of Public Goods Theory for Interactive Communication Systems}

Four issues in public goods theories have important implications for the present extensions to interactive communication systems. These include jointness of supply, impossibility of exclusion, coalition formation, and privatization.

\section{Jointness of Supply}

Jointness of supply in a connective good ideally means that each member enjoys the freedom to communicate with any other member such that one member's use of the system does not reduce the accessibility of the system to any other. Also, use of a system must not deplete its capabilities over time thereby hindering the free communicative capabilities of other users. For example, connectivity is provided through an electronic mail system when all members of the relevant public have accounts on 
the system, can access each other at any time, and use of the system does not somehow diminish system resources.

However, jointness of supply can be limited when "crowding" appears. This occurs when there are limits to physical connection on the system (i.e., it does not have the capability to support the volume of traffic) or when users access the system in such a way that others are somehow prohibited from free access. For example, a busy signal on a telephone system prevents access and hinders the capability to communicate with a chosen other. Also, when the volume of telephone users calling a locale is too heavy, all available lines may be in use, again prohibiting access. Importantly, these technical and user-related limits are being addressed in communication system design. In the telephone example, responses to such limitations include call-waiting features that permit interruption of a busy line and fiber optic cable that carries a much higher volume of traffic than copper wire.

Jointness of supply in a communal public good implies that information is simultaneously accessible to all users in the relevant public. In addition, updates by different users must occur promptly so that information remains current. Finally, one's use of information must not degrade its worth for subsequent users. For example, the public good of communality might be realized with a networked database to which all relevant users have at least "read" access and to which updates are made in real time. In this way users know where to find information, can access it jointly, retrieve current data, and presumably, do not corrupt information contained in the database.

\section{Impossibility of Exclusion}

Potential users are sometimes involuntarily excluded from the physical connectivity of a communication system. For instance, the system infrastructure may not be available in one's area (particularly common in the early stages of a new communication system) or financial constraints may limit access to the physical means of connection (telephone handset, computer, computer modem, etc.). On the other hand, exclusion from social connectivity is largely a matter of choice on the part of the user, once physical connectivity is established. For example, if people choose not to answer the telephone or use it to make calls, they are functionally excluded from the public good of social connectivity via the telephone system.

However, there need not be universal use for an interactive communication system to succeed, although the system will not be ideal in terms of universal access. First, the multifunctional aspects of some systems may support auxiliary communication functions with less than full or even optimal connectivity. Although social connectivity may not be supported in strict terms, the return to nonadopter status postulated by Markus (1990) may not take place. Second, interdependence of the medium with other media may support a multimedia system in which the availability of complementary media makes experimentation with a new 
medium feasible. Finally, full connectivity may fail among members of one community, but active communication may still support connecting a redefined, smaller community base. This allows for alternative interpretations of what constitutes use and communication in the system and what constitutes a public linked through connectivity.

Exclusion from a communal good in an information system can occur when an individual does not meet one or more of several criteria, such as membership in a particular organization or faction, possession of pertinent information for contribution, or need for the information in a given database, to name a few. In addition, if a database system has security features or other potential constraints that allow selective point-to-point transfer of information (direct connectivity), then the system provides the possibility of exclusion from the communal good with respect to selected information. Indeed, in interactive communication systems it is difficult to conceive of a system from which certain users within a potential public may not be excluded from the connective or communal good on some grounds.

Free-rider phenomenon. In a point-to-point communication system free riders on physical connectivity are those who use the system but are not active in obtaining and maintaining the system infrastructure. Schmitz, Rogers, Phillips, and Paschal (1993) described a homeless man who accessed a public electronic network via a terminal at the public library. As a nontaxpayer this individual is free riding on the connectivity of the physical system without contributing to its establishment or maintenance. However, he is not a free rider for social connectivity due to the fact that his very use of the system constitutes a contribution to social connectivity.

For communal information goods free riders are those who fail to contribute sufficiently to the jointly held body of information while continuing to enjoy its benefits. For example, the residents of a neighborhood wish to obtain the right to remodel local homes that are located in a geologically unstable area. To make the case homeowners need to collect geotechnical information about the neighborhood. Some homeowners could refuse to provide geologic data about their specific properties but still use the overall neighborhood database assembled by others to make the necessary arguments to obtain their own remodeling permits. Or, in the case of a law enforcement consortium, some agencies use information in the database obtained by other agencies' informants, but they refuse to contribute any information obtained from their own informants for fear of compromising their identities.

It may seem at first that it would be logical, and possible, to exclude all free riders from the public goods provided by interactive communication systems. However, in some situations it will be difficult to do so. For communal information goods some persons free ride by failing to contribute to the jointly held body of information. The identification and removal may be problematic when the information value of contri- 
butions is difficult or costly to assess. Individuals may simply contribute low-quality information that is difficult to see or trace promptly and reliably. Free riders may also persist when overall patterns of contributions are irregular, making it difficult to identify when an individual has ceased contributing. Removal of communal free riders may not even be desirable in those situations where their use of the system benefits the public as a whole. For example, a law enforcement agency that is "out of the loop" may take action that unknowingly endangers the personnel or operations of other agencies. Thus, it is desirable to keep even noncontributors informed to protect the safety of all officers.

For point-to-point communication systems the social contributions made by free riders on the physical connections may potentially enrich the social interactions on the system. In the instance of the homeless free rider mentioned earlier, his electronic mail messages to city officials and to others may be seen as a contribution to the democratic and social processes of the city and its users even while he free rides on the physical connectivity supplied by the public library. Another example of free riders on physical connectivity in a communication system is seen with the Lifeline service mandated through the telephone company in some areas. This service provides physical connection to those who cannot afford basic telephone service. The overall society is seen to benefit from the ability to link all members of the community to each other.

\section{Coalition Formation in the Realization} of the Public Good

Public goods theory proposes that sometimes a subset of the relevant public can contribute the full amount of resources to provide the public good (Oliver, Marwell, \& Teixeira, 1985). The key issue is motivating a sufficient number of resource-rich entities to secure the good for the benefit of the whole. A community park, for example, can be donated by a single individual. Hardin (1982) refers to such people as "political entrepreneurs" who "for their own career reasons, find it in their private interest to work to provide collective benefits to relevant groups" (p. 35). However, if individuals are resource-poor relative to the resources needed to create the good and no small subset can reasonably provide the good for all, wide participation of the public may be required to secure it. In either case, the good that is provided is indivisible and available to all, regardless of who contributed.

One implicit assumption of Connolly and Thorn's (1990) conceptualization of a discretionary database is that most or all members of the public hold some information of value to others such that information resources are widely distributed. Thus, a small coalition generally cannot create the full database for the whole. However, it is possible that a viable coalition of participants may contribute to and use the joint data. If the players with the most critical resources are part of this coalition, the system would be viable, although not ideal, since there likely would be some free riders. Interventions to induce contributions might be re- 
quired but only until the adoption base reached a critical point, at which time adoption and use of the system would be self-reinforcing (Markus \& Connolly, 1990).

It is also entirely possible that there will be subsets of people with common interests who can create a viable subset of the database that will benefit themselves but not the rest of the public. This can happen under two conditions. First, if information resources are unequally distributed the information rich may coalesce to create a database accessible only to themselves. For example, this might be accomplished through special coding of data or through imposition of exclusionary security codes for accessing data. Second, if information needs vary substantially across people such that some types of information are useful to only some members of the public, then subsets of people with common interests may work to create that portion of the database that is of most interest to them but ignore that part that is of interest to others. In either case, the net effect is the division of the public good, creating a partial public good that benefits different portions of the public selectively. Impossibility of exclusion is not fully obtained.

Viable coalitions can arise for connectivity in interactive communication systems in much the same way as for communality in discretionary databases. A subset of users may take the initiative to create the infrastructure that permits anyone in the public to access and use the system. Also, a viable coalition of users may thrive in the absence of full social connectivity. For example, in many electronic mail systems a subset of users interacts with each other even though others in the relevant public do not use the system that is available to them. Communication to nonusers occurs, if at all, through other communication media. The availability of other media to reach nonusers (e.g., universal access through the telephone) reduces the need for full connectivity on the electronic mail system. In this sense, then, the substitutability and complementarity of media contribute to the viability of subcommunities.

Viable coalitions could take the form of individuals with common interests communicating with each other but not with other members of the public, such as in distribution lists. Coalitions could also restrict access to themselves in ways similar to those of a database coalition. For example, a communication coalition could restrict distribution of its electronic mail addresses just as some individuals choose not to list their telephone numbers. A more cumbersome alternative is to fail to read and respond to communications from members of the public outside the coalition, thus becoming nonaccessible. Again, pure jointness of supply can be imperiled and impossibility of exclusion obtains only within the respective coalitions.

\section{Privatization of the Public Good}

Several findings from game theory, theories of rational decision making, and experimental tests of collective action theories suggest another aspect of public goods. The prisoner's dilemma (Hardin, 1982) stipulates that 
in single instances of the game people act in narrow self-interest. With multiple iterations, however, people tend to act in tacit collusion, maximizing their returns by a process of cooperation (Flood, 1958). When group size is increased, individual strategies return to self-interest and away from the public good (Hardin, 1982). Olson (1965) found this trend in his studies of collective action, where he contended that "the larger a group is, the farther it will fall short of obtaining an optimal supply of any collective good, and the less likely that it will act to obtain even a minimal amount of such a good. In short, the larger the group, the less it will further common interests" (p. 36). In a test of Olson's hypotheses, Sweeney (1973) found that the public good in large groups was most effectively provided for when a private good existed, the realization of which also contributed to the public good. That is, "a large group can only achieve its public goal as a 'by-product' of providing private goods" (p. 278). A large group may increase the likelihood of creating a larger public good by dividing into smaller coalitions. Several factors assist small groups in this process: (a) social identification is likely to be stronger, (b) members of smaller groups are better able to monitor and punish violation of the norms, and (c) members can more readily see the positive value of their own contributions (Baron, Kerr, \& Miller, 1992, p. 123).

If private goods were entirely self-interested, however, the wider public good would fail. In order for private goods to contribute effectively to the public good, coordinating factors must produce a wider public good from more narrow private goods. One strategy is to use a central authority to explicitly guide and coordinate private goods toward a wider public good. Such authority has both the perspective to see and the power to enact the coordination of private goods in order to realize the public good. Governmental regulation of telecommunication industries exemplifies such a strategy. A second strategy would be to create a lateral coordinative mechanism to ensure that subsets seeking private goods coordinate their activities in such a way that their goals serve to form the component parts of a broader public good. Through these mechanisms several private goods may complement one another toward the formation of the public good. ${ }^{3}$ Voluntary coordinative groups such as international standards-setting consortia for telecommunications transmission serve this role for supporting physical connectivity.

Private goods, coalitions, and hybrid goods. When private goods coexist effectively with public goods, hybrid goods are created. Separate coalitions formed by users are by definition private in relation to the broader public. The basic model of the hybrid communal good is a joint database that provides all users access to a set of information in a common pool, while some subsets of users share sets of additional information as private goods. Whether coalitions internally share information in a communal fashion is a separate matter. If they do, of course, public goods theory applies to them individually as smaller, distinct publics residing 
within the whole. Hybrids may at times be socially desirable for reasons already discussed: selective relevance of information, unpredictabilities about who might need specific information, relative costs, and effective enforcement of social norms.

Hybrid connective goods are illustrated by coalitions that send fax transmissions among themselves within the fully connected telephone network. Coalitions exist among those who have the resources of a fax machine and who are willing to restrict distribution of the telephone number of their separate fax lines to a certain subset of persons. In effect, they open their fax gateway only to a select group of persons. A second example is a police department that maintains an 800 (toll-free) number for its staff to call headquarters for instructions in the case of an emergency. Access to connectivity through the 800 subnetwork is restricted to police employees as a coalition.

Multifunctional hybrid goods seek to combine the strengths of connective and communal benefits in the context of both public and private goods. These goods flow from systems that offer one or more joint databases and direct connections in such a way that the entire relevant public is included in one but not the other. One basic model of this system is a single joint database that all users access, combined with selectively connected subsets of users. One example given earlier is a computer system that pools accounting data for all users in a database while only a small portion of the users are socially connected by electronic mail. The second basic model of a system providing a multifunctional hybrid good is a fully connected network where subsets of users communally share multiple, discrete pools of information. For example, database servers make it easier for coalitions of directly connected users to share data communally. Partial connection and communion exist within the context of full connectivity and one or more joint databases.

\section{Summary and Conclusion}

We have argued that two key dimensions underlie public goods in interactive communication systems: connectivity and communality. Connectivity exists when members of a public are linked through point-to-point communication. A key concern for the connective good is that its realization depends not only on physical infrastructure but also on social connectivity, which requires ongoing contributions in the form of regular system use rather than one-time investments. For social connectivity there can be no free riders because use is equivalent to contribution.

Communality is realized when members are linked through shared information. Communal goods from database systems help with the problems of (a) predicting which specific others could benefit from particular information, (b) determining what specific information will benefit specific others, and (c) promoting the synergy that can arise from combining disparate information in a communal database. It may also 
alleviate some of the technical and social costs of full point-to-point connectivity. When potentially important information is widely distributed across members of the community, each user controls a key gateway to development of the system. Thus, the costs and benefits to users of both communal and connective goods are dependent on the use of the good itself. This is a type of complexity not found in purely physical goods such as public parks.

The convergence of computing and telecommunications has supported a third category of good, that we label multifunctional, which combines aspects of communality and connectivity. Many of these multifunctional public goods are also interdependent with private goods. This interdependence provides more opportunities for the development of critical mass through crossover effects. However, the assumption of impossibility of exclusion upon which public goods theories rest is imperiled. Impossibility of exclusion is not fully attainable because communal databases can be divisible into private databases for coalitions, and multimedia communication systems can support a vast array of mediaspecific subcommunities. Impossibility of exclusion is also threatened by the practical divisibility of the physical system (nodes can be excluded) and by the need for regular system use by the public in order to realize a connective or communal good in full.

Heterogeneity of interests and resources can increase the likelihood of obtaining a connective or communal information good when it implies that either some users will be highly motivated to ensure provision of the good for all (excluding social connectivity, which can only be provided by the individual users themselves) or some users will have sufficient resources to provide incentives for the public to contribute to the good. However, heterogeneity can inhibit realization of a public good when there are constraints on contributions such that those with the greatest resources are not permitted to contribute more than an equal share and those with low resources cannot contribute the full amount of their equal share. Heterogeneity can also lessen the likelihood of achieving the good if differences in interests and resources divide the public. A good that is accessible to the full public will not be realized if resource-rich members create goods from which resource-poor individuals are involuntarily excluded. For example, this can occur when nodes are excluded from a connective good because select individuals cannot afford the user fees, or when a coalition denies outsiders access to its communal information. In addition, communality represents a public good only where members of the public consider the pooling of information to be beneficial. If heterogeneity takes the form of members holding qualitatively different information interests, some will hold information whose relevance is relatively narrow. Subsets of members who share common, narrow interests are likely to prefer connective or hybrid goods over simple communality if they wish to share such information privately.

Size of community appears to be related to realization of the good in 
complex ways. Two of the most important factors upon which this relationship depends are noticeability of contribution and the degree to which there is high jointness of supply.

\section{Suggestions for Future Research}

The theoretical extensions in this article provide an extensive platform for future theoretical and empirical work. Many areas should be developed in the conceptualization of interactive communication systems as supporting connective and communal public goods. One is the utility of information as a public rather than private good. For example, the value of different types of private information will change in complex ways in the transition from private to public good. Information costs, benefits, and value will also change in relation to the context of the information and to its manipulation in the creation of new meanings.

A second area for development concerns the implications of the connective and communal dimensions in multifunctional goods. Further analysis would examine interactive communication systems to identify the implications of each function for these public goods. Focus on the functions of connectivity and communality also integrates study of the processes and effects of different physical systems with common functions.

A third area to research is the dynamics of these systems over time (Monge, 1990, 1993), in line with changes in technology, user compositions, and alternative sources of connectivity and communality. The evolving character of systems is particularly important as it affects the relative prominence of connectivity and communality in multifunctional goods and the role of private goods for hybrids. Another key temporal issue in database systems is the perishability of information. Information in a database changes in value over time, is subject to obsolescence, and requires continual updating by contributors. The implications of this perishability for communal goods must be analyzed in the context of public goods theory.

Fourth, it is also important to view connectivity as a good provided through composite media systems. These systems are higher order aggregates of individual media, whether they support unifunctional, multifunctional, or hybrid goods. Composite systems include an array of connective capabilities available to persons at any one time through different media. For example, most persons have access to a telephone, the postal system, express mail, community forums, and so forth. The connectivity provided in combination by these and other options creates conditions for substitutability and complementarity of media. Therefore, the configuration of the composite media system affects the feasibility of developing new connective public goods. Also, because configurations will change with technological developments, the composite affects the likelihood that an adopter will return to nonadopter status for any medium that is a component. For example, the rapid disappearance of the pony express was linked to technological developments in communication that provided a more efficient substitute for the connectivity that system provided. 
Fifth, attention should be directed toward communication as a critical process that supports or inhibits the development of any public good. Public goods formulations have largely ignored communication among the potential public as a factor in developing a collective good. The group size claims exemplify the problem. Research based on $n$-player iterated prisoner's dilemma games has shown that smaller groups are more successful. Large groups tend to fail because either it is more difficult to track individual strategies or it is easier to hide treachery. When communication is inserted into the game, the entire logic is called into question. Research by Dawes (1991), Hage (1984), and Marwell and Oliver (1984) demonstrated that if people are able to communicate and cooperate from the start, the social dilemma of free riding may be reduced or avoided. These findings suggest that interactive communication systems should be studied not only in the context of connective and communal public goods but also as means to support communication processes in the development and maintenance of public goods generally. If individuals are able to negotiate solutions effectively among themselves, then their ability to arrive at solutions that emphasize individual and collective goals simultaneously is vastly improved. If such negotiation is possible, the autonomy, privacy, and self-determination of the individual may be combined effectively with collective outcomes.

Finally, the role of communication among contributors also points to important policy considerations that derive from an understanding of interactive communication systems as public goods. Policy analysts can formulate strategies for allocation of resources or for regulatory actions that influence the likelihood that the public good will be realized. For example, knowledge of collective action premises for interactive communication systems can help to determine the nature, degree and targets of government investments in communication infrastructure for the information superhighway. Appropriate investments can be targeted to accelerate the pace at which advanced communication and informationsharing capabilities are broadly accessible and widely employed. And, as Valente (1993) pointed out, once critical mass is realized, these incentives can be reduced because adoption and use will be self-sustaining (Markus \& Connolly, 1990). Efficient allocation of scarce resource investments to increase adoption and use can be realized through a careful understanding of the dynamics of public goods and the identification of the inflection point beyond which incentives are no longer necessary or cost effective.

Janet Fulk is associate professor and associate director; Andrew Flanagin, Michael E. Kalman and Timothy Ryan are doctoral students, and Peter Monge is professor in the Annenberg School for Communication, University of Southern California, Los Angeles, CA 90089.

The preparation of this article was supported by a grant from the National Science Foundation (No. SBR-922537), Janet Fulk and Peter Monge, coprincipal investigators. The authors thank Claire Parnassa, Suzanne Rumsey, David Waterman, and Don Ellis and anonymous reviewers for Communication Theory for belpful suggestions on an earlier version of this article. 
1 Head (1972) noted that a subset of public goods termed public bads is defined by the reciprocal characteristic of "impossibility of rejection" in that people cannot escape it (e.g., polluted air).

Olson (1965) described the production function as the relationship between cumulative contributions toward the collective good and the level of collective good realized. A decelerating function represents situations in which the level of collective good increases less quickly as contributions accumulate (decreasing marginal returns). Those persons who help to establish the collective good with early contributions have the greatest effect on its level. For example, the first persons to organize a beach cleanup by fixing a date, getting city permits, and publicizing the event have the most likelihood of ensuring its success. Subsequent contributions of trash receptacles and prizes for volunteers increase the likelihood of success, but the incremental effect is smaller.

By contrast, an accelerating production function represents situations in which later contributors have a relatively greater effect on the cumulative level of the good. For example, the first road that connects two points in an underdeveloped area of the world benefits only those who have direct access to it. As new roads get linked directly to earlier ones, more people can travel more places. Each new link expands the possibilities for travel geometrically and magnifies the benefits.

Initial contributions have more effect in the decelerative case, but the good may not be fully realized since the benefits of later contributions are so small. In the accelerative case, collective action is difficult to realize because the benefits to initial contributors are so small. However, if initial contributions can be attained they tend to snowball, increasing the likelihood that the collective good will be realized fully. Markus (1990) proposed that the production function for interactive media will be accelerating, because each community member is most likely to contribute when the payoff is greatest-after others are already connected and reachable through the medium.

3 Samuelson (1954), however, rejected such an argument on the grounds that aggregated private goods are not equivalent to public goods per se.

\section{References}

Badaracco, J. L., Jr. (1991). The knowledge link: How firms compete through strategic alliances. Cambridge, MA: Harvard Business School Press.

Baron, R. S., Kerr, N. L., \& Miller, N. (1992). Group process, group decision, group action. Pacific Grove, CA: Brooks/Cole.

Barry, B., \& Hardin, R. (1982). Rational man and irrational society. Beverly Hills, CA: Sage.

Bormann, E. G. (1983). Symbolic convergence: Organizational communication and culture. In L. L. Putnam \& M. E. Pacanowsky (Eds.), Communication and organizations: An interpretive approach (pp. 99-122). Beverly Hills, CA: Sage.

Bradley, S. P., Hausman, J. A., \& Nolan, R. L. (1993). Globalization, technology, and competition: The fusion of computers and telecommunications in the 1990s. Cambridge, MA: Harvard Business School Press.

Bureau of Justice Statistics (1992, December). Drugs, crime, and the justice system. Washington, DC: Bureau of Justice Statistics, NCJ-133652.

Chaiken, J., Chaiken, M., \& Karchmer, C. (1990). Multijurisdictional drug enforcement strategies: Reducing supply and demand. Washington, DC: National Institute of Justice.

Chamberlain, J. (1974). Provision of collective goods as a function of group size. Political Science Review, 68, 707-713.

Cohen, M. D., March, J. D., \& Olsen, J. (1972). A garbage can model of organizational choice. Administrative Science Quarterly, 17, 1-25.

Connolly, T., \& Thorn, B. K. (1990). Discretionary databases: Theory, data and implications. In J. Fulk \& C. Steinfield (Eds.), Organizations and communication technology (pp. 219-234). Newbury Park, CA: Sage.

Cook, K. S. (1991). The microfoundations of social structure: An exchange perspective. In J. Huber (Ed.), Macro-micro linkages in sociology (pp. 29-45). Newbury Park, CA: Sage.

Dawes, R. M. (1991). Social dilemmas, social self-interest, and evolutionary theory. In R. M. Coughlin (Ed.), Morality, rationality, and efficiency: Perspectives on socioeconomics, 1990 (pp. 17-40). New York: Sharpe.

Ekeh, P. P. (1974). Social exchange theory: The two traditions. Cambridge, MA: Harvard University Press. 
Flood, M. (1958). Some experimental games. Management Science, 5, 5-26.

Fulk, J., Von Glinow, M. A., \& Rogers, E. M. (1988). Managing change through communication technologies in third world countries. Journal of Change Management, 1, 21-37.

Habermas, J. (1979). The public sphere. In A. Mattelart \& S. Siegelaub (Eds.), Communication and class struggle (Vol. 1, pp. 198-201). New York: International General.

Hage, J. (1974). Communication and organizational control: Cybernetics in bealth and welfare organizations. New York: Wiley.

Hardin, R. (1982). Collective action. Baltimore: Johns Hopkins University Press.

Head, J. G. (1972). Public goods: The polar case. In R. M. Bird \& J. G. Head (Eds.), Modern fiscal issues: Essays in honour of Carl S. Shoup (pp. 7-16). Toronto, Ontario: University of Toronto Press.

Markus, M. L. (1990). Toward a "critical mass" theory of interactive media. In J. Fulk \& C. Steinfield (Eds.), Organizations and communication technology (pp. 194-218). Newbury Park, CA: Sage.

Markus, M. L., \& Connolly, T. (1990, October). Why CSCW applications fail: Problems in the adoption of interdependent work tools. CSCW Proceedings '90, 371-380.

Marwell, G., \& Oliver, P. (1984). Collective action theory and social movements research. Research in Social Movements, Conflict and Change, 7, 1-28.

Marwell, G., \& Oliver, P. (1993). The critical mass in collective action. New York: Cambridge University Press.

McQuail, D. (1987). Functions of communication: A nonfunctionalist overview. In C. R. Berger \& S. H. Chaffee (Eds.), Handbook of communication science (pp. 327-349). Newbury Park, CA: Sage.

Monge, P. R. (1990). Theoretical and analytical issues in studying organizational processes. Organization Science, 1, 406-430.

Monge, P. R. (1993). (Re)Designing dynamic organizations. In G. P. Huber \& W. H. Glick (Eds.), Mastering organizational change (pp. 323-345). Oxford, England: Oxford University Press.

Oliver, P. E. (1993). Formal models of collective action. Annual Review of Sociology, 19, 271-300.

Oliver, P., \& Marwell, G. (1988). The paradox of group size in collective action: A theory of the critical mass II. American Sociological Review, 49, 601-610.

Oliver, P., Marwell, G., \& Teixeira, R. (1985). A theory of the critical mass I: Interdependence, group heterogeneity, and the production of collective action. American Journal of Sociology, 91, 522-556.

Olson, M., Jr. (1965). The logic of collective action. Cambridge, MA: Harvard University Press.

Panko, R. (1984). Electronic mail: The alternatives. Office Administration and Automation, 45(6), 37-43.

Rafaeli, S., \& LaRose, R. J. (1993). Electronic bulletin boards and "public goods" explanations of collaborative mass media. Communication Research, 20, 277-297.

Samuelson, P. (1954). The pure theory of public expenditure. Review of Economics and Statistics, 36, 387-389.

Schmitz, J., Rogers, E., Phillips, K., \& Paschal, D. (1993, May). The Public Electronic Network (PEN) and the Homeless in Santa Monica. Paper presented at the annual meeting of the International Communication Association, Washington, DC.

Sirbu, M. A. (1992). The struggle for control within the telecommunications networks. In H. M. Sapolsky, R. J. Crane, W. R. Neuman, \& E. M. Noam (Eds.), The telecommunication revolution (pp. 140-148). New York: Routledge.

Stolte, J. F. (1987). The formation of justice norms. American Sociological Review, 52, $778-787$.

Sweeney, J. W., Jr. (1973). An experimental investigation of the free-rider problem. Social Science Research, 2, 277-292.

Valente, T. W. (1993). Diffusion of innovations and policy decision-making. Journal of Communication, 43, 30-45.

Von Neumann, J., \& Morgenstern, O. (1944). Theory of games and economic behavior. Princeton, NJ: Princeton University Press. 